\title{
Segmentação de Vértebras e Diagnóstico de Fraturas em Imagens de Ressonância Magnética Utilizando U-Net 3D e Deep Belief Network
}

\author{
Anderson Matheus Passos Paiva ${ }^{1}$, João Otávio Bandeira Diniz ${ }^{1}$, \\ Aristófanes Corrêa Silva ${ }^{1}$, Anselmo Cardoso de Paiva ${ }^{1}$ \\ ${ }^{1}$ Núcleo de Computação Aplicada - Universidade Federal do Maranhão (UFMA) \\ Campus Bacanga - São Luís - MA \\ \{andersonpaiva, joao.bandeira, ari, paiva\}@nca.ufma.br
}

\begin{abstract}
Lumbar pain is a common reason for clinical visits and magnetic resonance imaging is frequently used in systems to support the diagnosis of spinal pathologies. Aiming to improve and automate this process, this study proposes the use of computational techniques for the segmentation of vertebrae in magnetic resonance imaging, with the purpose of performing further analysis about pathologies in the spine. To achieve this goal, two Deep Learning architectures are used: U-Net for 3D segmentation and Deep Belief Network for the classification of vertebrae with rupture or not. The results show that U-Net is promising to localize the vertebra region, obtaining an average Dice Coefficient value of $89,51 \%$, thus overcoming several important studies focused on the problem. Classification was also efficient, with values of $94.38 \%$ for accuracy and $88.8 \%$ for sensitivity.
\end{abstract}

Resumo. A dor lombar é uma razão comum para visitas clínicas e o exame de ressonância magnética é frequentemente utilizado em sistemas de apoio a diagnóstico de patologias na coluna. Visando aprimorar e automatizar esse processo, este estudo propõe o uso de técnicas computacionais para a segmentação de vértebras em imagens de ressonância magnética, com o objetivo de realizar posteriores análises acerca de patologias na coluna. Para este fim, são utilizadas duas arquiteturas de Deep Learning: a U-Net para a segmentação em $3 D$ e a Deep Belief Network para a classificação de vértebras que apresentam ruptura ou não. Os resultados obtidos mostram que a U-Net é promissora em localizar a região da vértebra, obtendo um valor de Coeficiente de Dice médio de $89,51 \%$, superando assim vários trabalhos importantes focados no problema. A classificação também se mostrou eficiente, com valores de 94,38\% para acurácia e $88,8 \%$ de sensibilidade.

\section{Introdução}

Problemas na coluna, em geral dor lombar, vêm se mostrando muito frequentes e sua ocorrência apenas aumenta ao longo dos anos [Freburger et al. 2009]. Cerca de $80 \%$ da população sofre de dor nas costas em algum momento de suas vidas e, embora felizmente a grande maioria se recupere sem intervenção médica ou cirúrgica, para outros esse tipo de problema persiste e pode interferir na vida cotidiana [ANA 2019]. 
Em geral, para patologias envolvendo as vértebras, são utilizados exames de raio $\mathrm{X}$, contudo um diagnóstico mais eficiente é realizado baseado em imagens de Tomografia Computadorizada (TC) e Ressonância Magnética (RMI). O risco de câncer decorrente da exposição à radiação em TC torna a RMI preferível em rotinas clínicas [Richards et al. 2010]. Além disso, imagens de raio X (que são 2D) e TC não revelam todas as patologias devido à baixa sensibilidade dos tecidos moles. Nesse caso, o uso de RMI não é apenas recomendável, mas necessário.

Patologias como escoliose (curvatura na direção anatômica esquerda-direita), fratura vertebral (vértebra comprometida) e espondilolistese (vértebra desalinhada) podem ser diagnosticadas a partir das formas, posições e orientações das vértebras; portanto, suas segmentações são um passo necessário para o diagnóstico. Fraturas, em especial, geralmente são causadas por estresse mecânico aplicado a um osso enfraquecido, tipicamente resultado de osteoporose. Na prática clínica, o diagnóstico é geralmente estabelecido com base na impressão qualitativa do especialista [Lenchik et al. 2004].

Nesse contexto, há uma abundância de métodos de segmentação para vértebras em imagens de TC, o que é mais fácil de ser feito devido às altas intensidades entre o osso cortical e o tecido mole adjacente [Zukić et al. 2014]. De fato, métodos para a segmentação 3D de vértebras em RMI são mais desafiadores. Por exemplo, em imagens de TC as bordas ósseas são as únicas que apresentam alta intensidade. Isso não acontece quando trabalha-se com RMI, onde bordas entre alguns tipos de tecidos e até dentro do próprio corpo vertebral podem ser evidenciados. Além disso, as intensidades não são homogêneas em imagens de ressonância, mesmo dentro de uma região (o centro de uma vértebra possui maiores intensidades e melhor contraste do que as regiões marginais). Imagens de TC possuem a escala de unidade Hounsfield (HU) [Brooks 1977], o que não existe em RMI.

Várias abordagens já foram utilizadas para a segmentação de imagens médicas em RMI, e existem trabalhos com grande contribuição envolvendo vértebras e coluna vertebral, desde métodos envolvendo técnicas mais básicas como thresholding (limiarização), como técnicas mais robustas que incluem: crescimento de região, modelos deformáveis, Graph-Cut, modelos estatísticos baseados em forma e, mais recentemente, Deep Learning ou aprendizagem profunda, que se refere a redes neurais com muitas camadas que extraem características de imagens. Abordagens de segmentação baseadas em aprendizagem profunda para RMI estão despertando interesse devido a sua capacidade de autoaprendizagem e generalização sobre grandes quantidades de dados [Akkus et al. 2017].

Dito isso, o objetivo deste trabalho é segmentar, de forma automática e utilizando o espaço tridimensional, vértebras em RMI, para realizar em seguida diagnóstico de vértebras fraturadas. Decidiu-se optar por utilizar técnicas de Deep Learning, pois estas possuem alta capacidade em extrair características e obter uma representação complexa e abstrata dos dados, além de que ainda há muito o que se explorar nessa área acerca de aplicações em imagens de coluna. Para isso, utilizou-se a U-Net, uma das principais redes neurais no que se refere a segmentação de imagens médicas, para a detecção de vértebras em três dimensões, e a Deep Belief Network para classificar vértebras em duas classes: com e sem a presença de fratura.

O método proposto mostra-se bastante relevante para a comunidade científica. 
É proposto um método completamente automático para auxiliar especialistas não só na segmentação das vértebras, mas também na classificação destas em vértebras com ou sem fraturas. Ainda, pode-se listar uma série de contribuições que o trabalho englobou: (a) um método de segmentação/detecção de vértebras automática baseada em duas U-Net para segmentar com mais eficiência dois tipos de vértebras; (b) inserção de informações 3D na U-Net, gerando resultados mais promissores quando trata-se de RMI; e (c) utilização de uma Deep Belief Network, classificando o resultado da segmentação das redes U-Net em vértebras com ou sem fraturas. Assim, afirma-se que o método proposto pode ser utilizado em grandes centros, com o intuito de não só auxiliar o especialista na segmentação das vértebras, mas também classificá-las em vértebras fraturadas.

Além desta seção de introdução, o trabalho ainda apresenta mais 4 seções. Na Seção 2, serão discutidos os trabalhos relacionados, os quais apresentam métodos tanto para segmentação quando classificação de vértebras. Os materiais e o método proposto neste trabalho serão descritos na Seção 3. A Seção 4 apresenta os resultados e discussões da aplicação do método. Por fim, na Seção 5, conclui-se este trabalho apresentando melhorias futuras para o método.

\section{Trabalhos Relacionados}

Na Seção 1, discutiu-se algumas classes de métodos mais utilizadas para a segmentação de vértebras em imagens de Ressonância Magnética. Agora, será feita uma breve discussão sobre os principais trabalhos já desenvolvidos acerca desse assunto.

Uma abordagem que obteve resultados promissores utilizou Active Shape Models (ASM) e foi proposta por Neubert et al. (2011). Nesse trabalho, além das vértebras, foram segmentados os discos intervertebrais. O método automático foi testado em uma base de 14 pacientes, com um total de 132 vértebras, e obteve um valor de Coeficiente de Dice (DSC) [Dice 1945] de 91\%. Aqui, a segmentação das vértebras de cada paciente levava em torno de $5 \mathrm{~h}$, além da base de dados apresentar apenas pacientes saudáveis.

Zukić et al. (2014) apresentou um método que detecta e segmenta vértebras com mínima intervenção do usuário, além de realizar diagnóstico automático para detectar escoliose, espondilolistese e vértebras fraturadas. Para isso, foi utilizado o método de Viola-Jones para detecção dos centros das vértebras e uma combinação de mapa de probabilidades contendo características de intensidades e bordas com Balloon Inflation Forces para a segmentação 3D das vértebras a partir de modelos deformáveis. Com o resultado da segmentação, foram deduzidas métricas para obter os diagnósticos. Apesar da segmentação não ter ultrapassado o valor de $80 \%$ de Coeficiente de Dice, o resultado obtido foi suficiente para realizar os diagnósticos de forma satisfatória, uma vez que foram encontrados apenas 5,5\% de falso-positivos.

Schwarzenberg et al. (2014) optou por um método baseado em grafos, pelo qual deu o nome de $\mathrm{Cube-Cut}$. O algoritmo gera um grafo direcionado com dois nós terminais, onde os nós do grafo correspondem a um subconjunto dos voxels da imagem em forma de cubo. Os pesos das arestas, que conectam cada nó a uma fonte $\mathbf{s}$ ou um sorvedouro t, representam a afinidade do voxel com a vértebra (fonte) e com o fundo (sorvedouro). Após a construção do gráfico, um corte mínimo é realizado para separar as duas regiões. O valor médio de DSC obtido foi de $81,33 \%$. O trabalho foca bastante na velocidade de execução do método, que foi executado em menos de um minuto. 
A ideia de utilizar um limiar para realizar a segmentação já foi explorada em alguns trabalhos, porém em combinação com outras técnicas. Em seu método, Chu et al. (2015) localizou automaticamente os corpos vertebrais para definir regiões de interesse (ROIs) para uma etapa de segmentação subsequente, onde uma Random Forest foi utilizada para estimar a probabilidade de cada pixel dentro das ROIs pertencer ao objeto ou ao fundo. Os resultados foram combinados com um mapa de probabilidades para segmentar o corpo vertebral via thresholding, conseguindo assim um DSC de 88,7\%.

Obtendo grande destaque na área, Korez et al. (2016) introduziu uma abordagem baseada em redes neurais convolucionais (CNNs) na segmentação da coluna em RMI. Seu método aliou ASM com mapas de likelihood das vértebras e alcançou um DSC médio de 93,4\%. Korez treinou e testou seu método em 23 pacientes, os mesmos utilizados no trabalho de Chu et al. (2015).

Levando em consideração trabalhos utilizando crescimento de região, destaca-se o de Egger et al. (2017), que utilizou um método baseado em autômato celular chamado GrowCut. Foi utilizada a implementação contida na ferramenta Slicer. O valor médio de DSC encontrado foi de $82,99 \%$.

Por fim, destaca-se o trabalho de Hille et al. (2018) que, assim como alguns trabalhos anteriores, também utilizou mapa de probabilidades em seu método. Foi utilizada uma abordagem baseada em level-sets, onde buscou-se estimar a curva que melhor representasse a vértebra. Contudo, além da inicialização do método ser manual, o DSC encontrado com essa abordagem foi de $86 \%$, inferior a outros resultados encontrados previamente.

É importante notar que o melhor resultado obtido dentre os trabalhos do estado da arte foi encontrado utilizando Deep Learning, o que aponta para a eficácia de métodos da área no problema em questão. Apesar disso, muitos métodos de aprendizagem profunda ainda não foram utilizados e, considerando que modelos de Deep Learning são poderosas ferramentas de aprendizado e extração de características, torna-se interessante investigar essa área.

Além disso, a maior parte dos trabalhos apresentam alguma limitação em relação a base utilizada ou ao método, seja utilizando uma base apenas com pacientes saudáveis, ou desconsiderando vértebras de determinada região, ou utilizando métodos semiautomáticos (métodos de crescimento de região, por exemplo, necessitam de inicialização manual). Nesse ponto, o trabalho de Zukić et al. (2014) leva vantagem, apesar de apresentar o menor valor de DSC.

Assim, o método aqui proposto busca superar essas limitações diante dos trabalhos expostos, além de realizar uma etapa adicional de classificação, o que não é feito pela maior parte dos trabalhos da área (novamente, dentre os trabalhos citados, apenas o de Zukić et al. (2014) realiza uma etapa de classificação).

\section{Materiais e Método}

O método proposto está, basicamente, dividida em quatro etapas. Os materiais utilizados no trabalho são apresentados na etapa de aquisição de imagens. A segunda etapa é a de pré-processamento, e nela descreve-se o método de correção de bias field. A segmentação das vértebras é a terceira etapa, onde utiliza-se duas redes U-Net para a região da vértebra 
ser obtida. Por fim, é apresentada a etapa de classificação. Nela, utiliza-se a saída da etapa anterior como entrada em uma Deep Belief Network, com o objetivo de detectar vértebras com fratura.

Uma etapa adicional é a de validação, que é discutida com mais detalhes na seção seguinte. A Figura 1 ilustra os passos do método.

\subsection{Aquisição de Imagens}

O foco desse trabalho está na segmentação de vértebras lombares e sacro, apesar de também ser aplicado em vértebras torácicas. A coluna vertebral humana é dividida em cinco regiões: coluna cervical (sete vértebras), coluna torácica (doze vértebras), coluna lombar (cinco vértebras) e as regiões sacral e coccígea. A coluna lombar ou parte inferior das costas é a terceira maior parte da coluna vertebral e está localizada abaixo da região torácica, consistindo de cinco vértebras separadas, denominadas sequencialmente de L1 a L5. A coluna lombar é projetada para ser forte e para proteger a medula espinhal altamente sensível e as raízes nervosas espinhais, mas, ao mesmo tempo, é flexível para proporcionar mobilidade [Mendieta 2016].

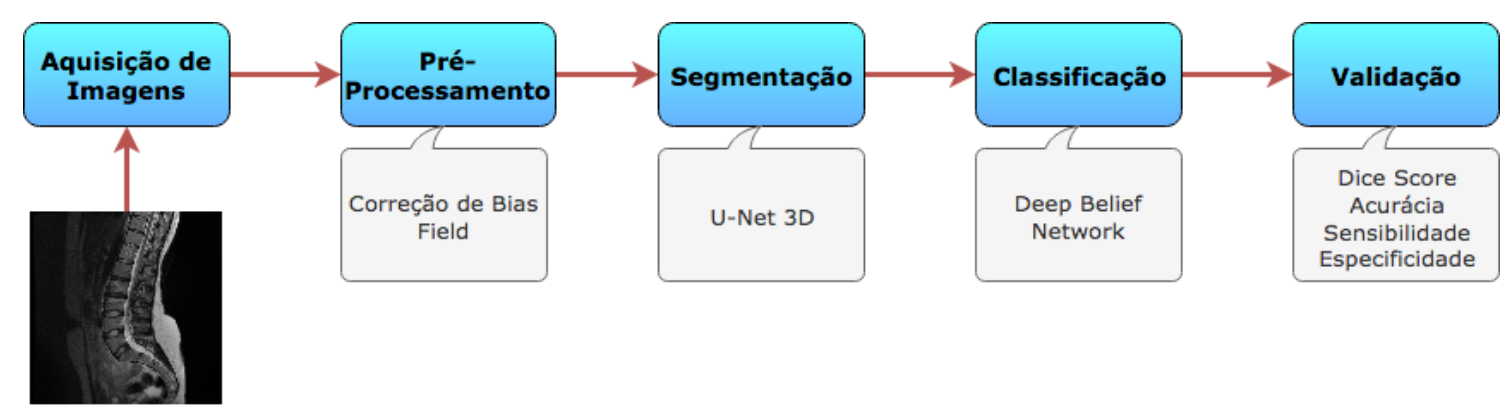

Figura 1. Etapas do método

Uma vértebra lombar típica pode ser descrita como uma estrutura óssea irregular com nomes específicos para essas irregularidades. Cada vértebra lombar pode ser subdividida em três regiões principais: corpo vertebral, pedículos e elementos posteriores. Os corpos vertebrais são conectados entre si pelos discos intervertebrais, e de L1 a L5 o tamanho dos corpos vertebrais aumenta, o que é um indicativo do aumento da carga para a vértebra lombar inferior. O corpo vertebral é perfeitamente moldado para lidar com essas cargas, pois é semelhante a uma caixa, com as superfícies superior e inferior substancialmente planas, e as superfícies anterior e lateral ligeiramente côncavas. Esse design de suporte de carga também é refletido na estrutura interna. A casca externa é feita de osso cortical ao redor de um núcleo ósseo esponjoso.

A base de imagens utilizada foi obtida de exames de 17 pacientes que foram disponibilizados pelo trabalho de Zukić et al. (2014) em formato DICOM, em um total de 160 vértebras com patologias ou não. Todos os exames possuem marcações de referência, e apresentam diversidade em tipos de vértebras, contrastes e vértebras saudáveis e patológicas.

O número de vértebras para cada paciente varia, e ao todo trabalhou-se com 13 tipos diferentes, sendo 7 da região torácica (de T6 a T12), 5 da região lombar (de L1 a L5) e uma da região sacral (S1). A Figura 2 ilustra uma fatia de um paciente do conjunto, 
exibindo ao lado a marcação do especialista em 9 vértebras: 3 torácicas (T10, T11 e T12), as 5 lombares e a vértebra sacral.

\subsection{Pré-Processamento}

Antes de realizar a etapa de segmentação, foi realizado a correção de bias field [Juntu et al. 2005] da base de imagens. Esse pré-processamento é necessário porque a intensidade do campo magnético não é perfeitamente homogênea em todo o volume de aquisição, e assim o mesmo tecido pode ter diferentes intensidades em diferentes partes da imagem. Dessa forma, encontra-se esse campo aditivo com o objetivo de tornar as intensidades e o contraste das imagens mais homogêneos.

A correção de bias field é ilustrada na Figura 3.
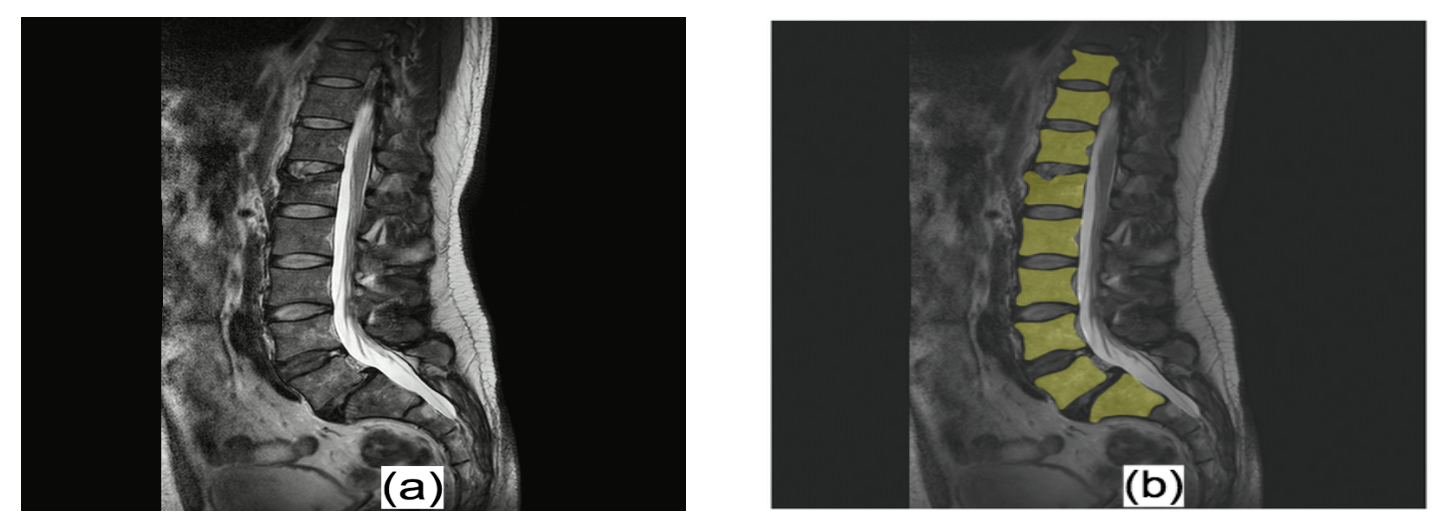

Figura 2. Exemplo de imagem da base. (a) Fatia do paciente F02 (b) Marcação do especialista para a fatia.

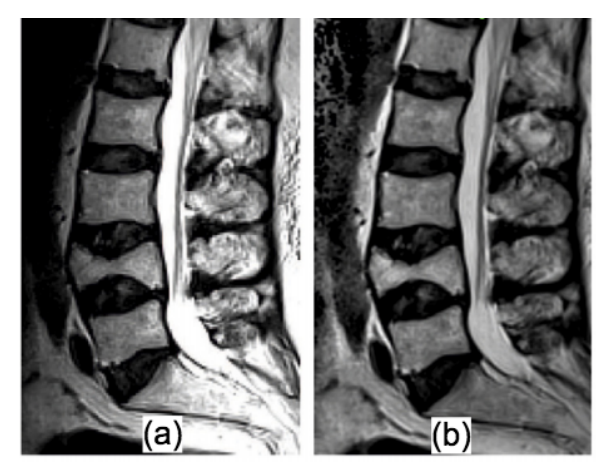

Figura 3. Correção de bias field. (a) Figura original (b) Com correção

\subsection{Segmentação das Vértebras com U-Net 3D}

A rede neural escolhida para a segmentação nesse trabalho foi baseada no trabalho de Lu et al. (2018) e é a U-Net [Ronneberger et al. 2015], que provou ser eficaz em tarefas de segmentação mesmo com dados limitados e tem sido usada para segmentação de imagens médicas. Contudo, no trabalho de referência, ela foi utilizada em 2D e com uma marcação manual simplificada, não correspondendo fielmente a uma máscara obtida por marcação de especialistas. 
Um problema apresentado e discutido no trabalho de Lu et al. (2018) se refere a vértebra sacral, uma vez que, ao utilizar apenas uma rede para a segmentação, os resultados ficavam abaixo do esperado devido a essa vértebra apresentar formato diferenciado das demais (trapezoidal em vez de retangular). Assim, a solução encontrada foi treinar uma U-Net para as vértebras torácicas e lombares e treinar outra U-Net apenas com as vértebras do sacro. Decidiu-se então adotar essa abordagem, porém a segunda rede foi treinada com a última vértebra lombar (L5) em conjunto com a sacral, visto que resultados preliminares apontaram que a U-Net também apresentou problemas em segmentar essa vértebra.

\subsection{Classificação das Vértebras com Deep Belief Network}

Para a etapa de classificação, utilizou-se a Deep Belief Network (DBN), baseada no trabalho de Liu et al. (2017), que utiliza essa rede neural para classificação de dígitos da base MNIST [LeCun and Cortes 2010] e cita que, recentemente, DBNs têm sido aplicadas à classificação de imagens, obtendo bons resultados. Essas redes são utilizadas para extrair características de imagens que, por fim, são classificadas utilizando algum classificador simples (por exemplo, uma função de ativação softmax).

Uma DBN pode ser definida como várias máquinas restritas de Boltzmann (RBM, do inglês Restricted Boltzmann Machine) empilhadas, na qual cada camada da RBM se comunica com as camadas anterior e posterior. Uma RBM, por sua vez, é uma rede neural estocástica baseada em energia. A arquitetura básica dessa rede consiste em uma camada visível e uma camada escondida. A camada visível representa os dados observados e a camada oculta tenta aprender a extrair características sobre esses dados.

Assim, são utilizadas as imagens resultantes do processo de segmentação como entrada para a DBN. Além disso, como o diagnóstico de fratura é feito para cada vértebra separadamente, considerou-se para a entrada da rede cada vértebra individualmente em vez de uma imagem completa de um paciente.

Por fim, obteve-se um modelo capaz de segmentar e classificar vértebras. A validação do método é discutida na próxima seção, onde utilizou-se o Coeficiente de Dice para avaliar a segmentação e as métricas de acurácia, sensibilidade e especificidade para avaliar a classificação.

\section{Resultados e Discussão}

Nesta seção serão apresentados e avaliados os resultados obtidos nas etapas de segmentação e classificação, em conjunto com discussões de resultados de outros trabalhos relevantes.

\subsection{Segmentação 3D}

Aqui, a arquitetura utilizada para as redes U-Net foi similar à do trabalho de Lu et al. (2018). As dimensões de entrada/saída da rede foram de 512x512x15, o que difere do trabalho de referência por trabalhar com o volume, e não uma fatia como entrada. Foram utilizados 5 módulos de max-pooling/up-pooling, além de 2 camadas convolucionais por módulo. A função de ativação final da rede foi a sigmóide e houve o uso de batch normalization. A taxa de aprendizado utilizada foi de 0,01 . 
Após o método proposto ter sido executado, foram obtidas as segmentações 3D para cada vértebra. A Figura 4 ilustra uma imagem de uma fatia (4a) ao lado do ground truth (4b) e o resultado da segmentação com a U-Net (4c).
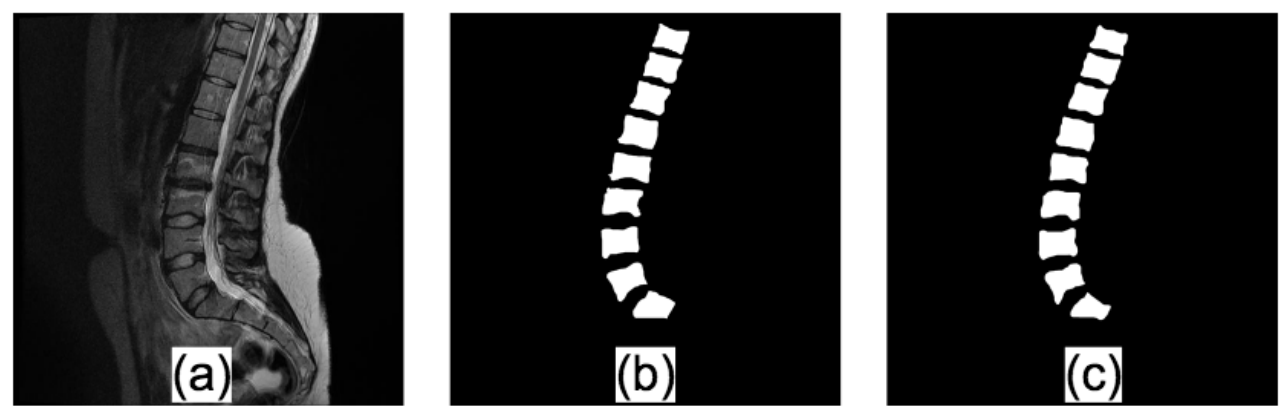

Figura 4. Resultado da segmentação 3D com a U-Net para uma fatia. (a) Fatia original (b) Marcação do especialista (c) Segmentação do método proposto

Os resultados obtidos foram validados com o Coeficiente de Dice (DSC), que é um índice utilizado para comparar a similaridade de duas amostras [Zou et al. 2004]. Ele se baseia na sobreposição de dois conjuntos, podendo assumir valores de 0 a 1 (valor ideal), e a sua fórmula é dada por:

$$
D S C(A, B)=\frac{2|A \cap B|}{|A+B|}
$$

Vale ressaltar que, dentre os trabalhos do estado da arte que foram analisados e possuem o mesmo objetivo, o DSC é a principal métrica para avaliar os resultados, por isso decidiu-se focar nesta métrica. Dito isso, o valor médio de DSC encontrado para a etapa de segmentação do método proposto foi de 0,8951. Para a U-Net responsável por segmentar as últimas duas vértebras (L5 e S1), o DSC encontrado foi de 0,8587, e para a outra rede o valor foi de 0,9035 . Uma análise objetiva e subjetiva dos resultados nos leva a entender que a U-Net ainda apresenta problemas em caracterizar as regiões de borda das vértebras.

Uma comparação com os trabalhos mais relevantes é feita na Tabela 1. Os métodos de Schwarzenberg et al. (2014) e Egger et al. (2017) também utilizaram imagens da base de Zukić et al. (2014), porém trabalharam com um número reduzido de vértebras (10 e 13, respectivamente). O método de Korez et al. (2016) trabalhou com a base disponibilizada por Chu et al. (2015) de 23 exames de pacientes saudáveis. O método de Hille et al. (2018) foi o que trabalhou com mais exames, utilizando as bases de Zukić et al. (2014), Chu et al. (2015) e mais duas bases privadas. Finalmente, o trabalho de Neubert et al. (2011) utilizou uma base própria de 28 exames de pacientes saudáveis que não se encontra mais disponível até então.

Com esses resultados, pode-se notar que o método proposto superou boa parte dos trabalhos anteriores levando em consideração o Coeficiente de Dice. Em comparação com os trabalhos de Neubert et al. (2011) e Korez et al. (2016), que apresentaram melhores resultados, percebe-se que aqui o método foi aplicado em uma base de imagens mais diversa, levando em consideração os tipos de vértebras (aqui foram utilizados 13 tipos em 
contrapartida aos outros métodos que foram aplicados em menos vértebras) e os contrastes utilizados (dois tipos em vez de um). Mesmo assim, o valor de DSC obtido é satisfatório e se equipara aos valores obtidos ao comparar segmentações manuais entre diferentes especialistas, que costumam variar entre $86 \%$ e $96 \%$, como comentado no trabalho de Zukić et al. (2014).

Outro fator que impacta diretamente nos resultados obtidos e que também é mencionado por Zukić et al. (2014) é o fato de que os trabalhos de Neubert et al. (2011) e Korez et al. (2016) foram aplicados em uma base de pacientes saudáveis. Pacientes com patologias mais graves, como vértebra fraturada, apresentam DSC mais baixos do que pacientes com vértebras saudáveis. Portanto, patologias naturalmente reduzem a taxa de detecção.

Tabela 1. Comparação de resultados de segmentação do método proposto com o estado da arte. * indica campo não especificado.

\begin{tabular}{|c|c|c|c|}
\hline Método & Vértebras & Contraste & DSC (\%) \\
\hline [Zukić et al. 2014] & T6-S1 & T1-,T2-w & 79,3 \\
\hline [Schwarzenberg et al. 2014] & L1-L5 & T2-w & 81,33 \\
\hline [Egger et al. 2017] & $*$ & T2-w & 82,99 \\
\hline [Hille et al. 2018] & T1-L5 & T2-w & 86,0 \\
\hline [Chu et al. 2015] & T11-L5 & T2-w & 88,7 \\
\hline [Neubert et al. 2011] & T8-L5 & T2-w & 91,0 \\
\hline [Korez et al. 2016] & T11-L5 & T2-w & 93,4 \\
\hline Proposto & T6-S1 & T1-,T2-w & $\mathbf{8 9 , 5 1}$ \\
\hline
\end{tabular}

\subsection{Classificação}

$\mathrm{Na}$ arquitetura da DBN, trabalhou-se com os resultados da segmentação da U-Net considerando as fatias de cada paciente, e não o volume. A dimensão de entrada da rede foi de 40x40, onde foi considerado cada pixel da imagem correspondendo a um neurônio da camada de entrada. Foi utilizada a proporção 80/20 para treino e teste.

Para a RBM, foram utilizadas duas camadas escondidas com 256 neurônios. A taxa de aprendizado foi definida como 0,05 e utilizou-se 10 épocas para treino das camadas de RBM e 100 épocas na etapa de backpropagation. Aqui, a função de ativação escolhida foi a ReLU. Novamente fez-se uso de batch normalization, porém com acréscimo de Dropout para cada camada de 0,2.

Após o treino da rede, das 160 imagens de teste, 151 foram classificadas de forma correta, obtendo-se assim uma acurácia de 94,38\%. A Tabela 2 ilustra melhor os resultados da classificação para as classes e as principais métricas são mostradas na Tabela 3. Com isso, podemos notar que a rede conseguiu identificar quase todas as vértebras danificadas.

Nota-se pelos resultados que a base se apresenta bem desbalanceada, com poucos exemplos de vértebras com fratura. Logo, a rede aprendeu melhor as características de vértebras sem fratura. Mas, se tratando de diagnóstico clínico, o mais importante é não deixar uma vértebra com fratura ser diagnosticada de forma errônea. Por esse lado, a DBN só errou na classificação de uma vértebra fraturada. 
Em comparação ao trabalho de Zukić et al. (2014), que também realizou diagnóstico de fraturas, a DBN obteve resultados inferiores, uma vez que o método de Zukić et al. (2014) conseguiu identificar todos os casos em que a vértebra realmente apresentava fratura e apresentou uma taxa menor de falso-positivos. Isso mostra que os resultados de diagnóstico estabelecidos usando nosso método ainda precisam ser otimizados.

Tabela 2. Matriz de confusão para a classificação das vértebras com DBN

\begin{tabular}{|c|c|c|}
\hline Real $\backslash$ Predição & Com fratura & Sem fratura \\
\hline Com fratura & 8 & 1 \\
\hline Sem fratura & 8 & 143 \\
\hline
\end{tabular}

Tabela 3. Métricas para a classificação das vértebras com DBN

\begin{tabular}{|c|c|c|c|}
\hline Métricas & Acurácia & Sensibilidade & Especificidade \\
\hline Valores & $94,38 \%$ & $88,89 \%$ & $94,7 \%$ \\
\hline
\end{tabular}

\section{Conclusão}

Neste trabalho foi apresentada um método automático para a segmentação de vértebras em RMI com o uso da U-Net 3D e classificação de vértebras quanto à presença ou não de fratura utilizando a Deep Belief Network. Os resultados obtidos nos mostram que a U-Net foi eficiente na etapa de segmentação em 3D, obtendo um Coeficiente de Dice de $89,51 \%$, o que é um valor satisfatório comparado ao estado da arte e às diferentes segmentações de especialistas. Outro fator que valida positivamente o resultado obtido é que a segmentação não é feita apenas em vértebras saudáveis, o que não acontece na maior parte dos outros trabalhos. Além disso, na etapa de classificação, a DBN conseguiu $88,89 \%$ de sensibilidade e, em diagnóstico clínico de fraturas, a sensibilidade é a métrica mais importante a ser considerada.

Para trabalhos futuros a respeito da segmentação com a U-Net, prevê-se ajustes de parâmetros e possivelmente o uso de outras arquiteturas de redes neurais e técnicas de Deep Learning. Na etapa de classificação, pretende-se investigar outros tipos de anomalias presentes na coluna, além da aplicação de técnicas de balanceamento ou pesos para as classes da base.

O presente trabalho foi realizado com apoio da Coordenação de Aperfeiçoamento de Pessoal de Nível Superior - Brasil (CAPES) - Código de Financiamento 001.

\section{Referências}

Akkus, Z., Galimzianova, A., Hoogi, A., Rubin, D. L., and Erickson, B. J. (2017). Deep learning for brain mri segmentation: state of the art and future directions. Journal of digital imaging, 30(4):449-459.

ANA (2019). Spine disorders, deformities and diseases. https: //ana-neurosurgery.com/areas-of-expertise/ spine-disorders-deformities-diseases/. Acesso em: 15 de fevereiro de 2019. 
Brooks, R. A. (1977). A quantitative theory of the hounsfield unit and its application to dual energy scanning. Journal of computer assisted tomography, 1(4):487-493.

Chu, C., Belavỳ, D. L., Armbrecht, G., Bansmann, M., Felsenberg, D., and Zheng, G. (2015). Fully automatic localization and segmentation of $3 \mathrm{~d}$ vertebral bodies from $\mathrm{ct} / \mathrm{mr}$ images via a learning-based method. PloS one, 10(11):e0143327.

Dice, L. R. (1945). Measures of the amount of ecologic association between species. Ecology, 26(3):297-302.

Egger, J., Nimsky, C., and Chen, X. (2017). Vertebral body segmentation with growcut: Initial experience, workflow and practical application. SAGE open medicine, 5:2050312117740984.

Freburger, J. K., Holmes, G. M., Agans, R. P., Jackman, A. M., Darter, J. D., Wallace, A. S., Castel, L. D., Kalsbeek, W. D., and Carey, T. S. (2009). The rising prevalence of chronic low back pain. Archives of internal medicine, 169(3):251-258.

Hille, G., Saalfeld, S., Serowy, S., and Tönnies, K. (2018). Vertebral body segmentation in wide range clinical routine spine mri data. Computer methods and programs in biomedicine, 155:93-99.

Juntu, J., Sijbers, J., Van Dyck, D., and Gielen, J. (2005). Bias field correction for mri images. In Computer Recognition Systems, pages 543-551. Springer.

Korez, R., Likar, B., Pernuš, F., and Vrtovec, T. (2016). Model-based segmentation of vertebral bodies from mr images with $3 \mathrm{~d}$ cnns. In Ourselin, S., Joskowicz, L., Sabuncu, M. R., Unal, G., and Wells, W., editors, Medical Image Computing and ComputerAssisted Intervention - MICCAI 2016, pages 433-441, Cham. Springer International Publishing.

LeCun, Y. and Cortes, C. (2010). MNIST handwritten digit database.

Lenchik, L., Rogers, L. F., Delmas, P. D., and Genant, H. K. (2004). Diagnosis of osteoporotic vertebral fractures: importance of recognition and description by radiologists. American Journal of Roentgenology, 183(4):949-958.

Liu, G., Xiao, L., and Xiong, C. (2017). Image classification with deep belief networks and improved gradient descent. In 2017 IEEE International Conference on Computational Science and Engineering (CSE) and IEEE International Conference on Embedded and Ubiquitous Computing (EUC), volume 1, pages 375-380.

Lu, J.-T., Pedemonte, S., Bizzo, B., Doyle, S., Andriole, K. P., Michalski, M. H., Gonzalez, R. G., and Pomerantz, S. R. (2018). Deepspine: Automated lumbar vertebral segmentation, disc-level designation, and spinal stenosis grading using deep learning. arXiv preprint arXiv:1807.10215.

Mendieta, J. B. (2016). An efficient and semiautomatic segmentation method for $3 \mathrm{~d}$ surface reconstruction of the lumbar spine from magnetic resonance imaging (mri). Master's thesis, Queensland University of Technology.

Neubert, A., Fripp, J., Shen, K., Salvado, O., Schwarz, R., Lauer, L., Engstrom, C., and Crozier, S. (2011). Automated 3d segmentation of vertebral bodies and intervertebral discs from mri. In 2011 International Conference on Digital Image Computing: Techniques and Applications, pages 19-24. 
Richards, P. J., George, J., Metelko, M., and Brown, M. (2010). Spine computed tomography doses and cancer induction. Spine, 35(4):430-433.

Ronneberger, O., Fischer, P., and Brox, T. (2015). U-net: Convolutional networks for biomedical image segmentation. In International Conference on Medical image computing and computer-assisted intervention, pages 234-241. Springer.

Schwarzenberg, R., Freisleben, B., Nimsky, C., and Egger, J. (2014). Cube-cut: vertebral body segmentation in mri-data through cubic-shaped divergences. PloS one, 9(4):e93389.

Zou, K. H., Warfield, S. K., Bharatha, A., Tempany, C. M., Kaus, M. R., Haker, S. J., Wells III, W. M., Jolesz, F. A., and Kikinis, R. (2004). Statistical validation of image segmentation quality based on a spatial overlap index1: scientific reports. Academic radiology, 11(2):178-189.

Zukić, D., Vlasák, A., Egger, J., Hořínek, D., Nimsky, C., and Kolb, A. (2014). Robust detection and segmentation for diagnosis of vertebral diseases using routine mr images. In Computer Graphics Forum, volume 33, pages 190-204. Wiley Online Library. 